\title{
LncRNA H19 Promotes Odontoblastic \\ Differentiation of Human Dental Pulp Stem Cells by Regulating miR-140-5p and BMP-2/FGF9
}

\section{Jialin Zhong}

Stomatology Hospital of Guangzhou Medical University

\section{Xinran Tu}

Stomatology Hospital of Guangzhou Medical University

\section{Yuanyuan Kong}

Stomatology Hospital of Guangzhou Medical University

\section{Liyang Guo}

Stomatology Hospital of Guangzhou Medical University

\section{Baishun Li}

Stomatology Hospital of Guangzhou Medical University

\section{Wenchao Zhong}

Stomatology Hospital of Guangzhou Medical University

\section{Ying Cheng}

Guangzhou Medical University

\section{Yiguo Jiang}

Guangzhou Medical University

Qianzhou Jiang ( $\sim$ jqianzhou@126.com)

Stomatology Hospital of Guangzhou Medical University https://orcid.org/0000-0003-2631-7527

\section{Research}

Keywords: long non-coding RNA, H19, human dental pulp stem cells (hDPSCs), dentinogenesis, odontoblastic differentiation, miR-140-5p

Posted Date: April 14th, 2020

DOl: https://doi.org/10.21203/rs.3.rs-16133/v2

License: (c) (1) This work is licensed under a Creative Commons Attribution 4.0 International License.

Read Full License 
Version of Record: A version of this preprint was published at Stem Cell Research \& Therapy on May 27th, 2020. See the published version at https://doi.org/10.1186/s13287-020-01698-4. 


\section{Abstract}

Background: Increasing evidence has revealed that long non-coding RNAs (IncRNAs) exert critical roles in biological mineralization.As a critical process for dentin formation, odontoblastic differentiation is regulated by complex signaling networks. The present study aimed to investigate the biological role and regulatory mechanisms of IncRNA-H19 (H19) in regulating the odontoblastic differentiation of human dental pulp stem cells (hDPSCs).

Methods: We performed IncRNA microarray assay to reveal the expression patterns of IncRNAs involved in odontoblastic differentiation. H19 was identified and verified as a critical factor by qRT-PCR. The gainand loss-of-function studies were performed to investigate the biological role of $\mathrm{H} 19$ in regulating odontoblastic differentiation of hDPSCs in vitro and in vivo. Odontoblastic differentiation was evaluated through qRT-PCR, Western blot and Alizarin Red S staining. Bioinformatics analysis identified that $\mathrm{H} 19$ could directly interact with miR-140-5p, which was further verified by luciferase reporter assay. After overexpression of miR-140-5p in hDPSCs, odontoblastic differentiation was determined. Moreover, the potential target genes of miR-140-5p were investigated and the biological functions of BMP-2 and FGF9 in hDPSCs were verified. Co-transfection experiments were conducted to validate miR-140-5p was involved in H19-mediated odontoblastic differentiation in hDPSCs.

Results: The expression of $\mathrm{H} 19$ was significantly up-regulated in hDPSCs undergoing odontoblastic differentiation. Overexpression of $\mathrm{H} 19$ stimulated odontoblastic differentiation in vitro and in vivo, whereas down-regulation of $\mathrm{H} 19$ revealed the opposite effect. $\mathrm{H} 19$ binds directly to miR-140-5p and overexpression of miR-140-5p inhibited odontoblastic differentiation of hDPSCs. H19 acted as a miR-140$5 p$ sponge, resulting in regulated the expression of BMP-2 and FGF9. Overexpression of $\mathrm{H} 19$ abrogated the inhibitory effect of miR-140-5p on odontoblastic differentiation.

Conclusion: Our data revealed that $\mathrm{H} 19$ plays a positive regulatory role in odontoblastic differentiation of hDPSCs through miR-140-5p/BMP-2/FGF9 axis, suggesting that $\mathrm{H} 19$ may be a stimulatory regulator of odontogenesis.

\section{Background}

Due to the strong self-renewal capacity and regenerative properties, the biological effects of mesenchymal stem cells (MSCs) have been widely acknowledged and their application on bone tissue engineering have made great progress ${ }^{[1]}$. As a promising type of adult stem cell, human dental pulp stem cells (hDPSCs) can proliferate and differentiate into odontoblasts and subsequently deposit reparative dentine as a stress reaction when the pulp tissue is subject to bacterial, chemical or physical stimulation ${ }^{[2]}$. Owing to the enormous proliferative capacity and multi-potentiality, hDPSCs are capable for adipogenic, osteogenic, dentinogenic, neurogenic differentiation under specific induction environments $^{[3]}$. Thus, the complex molecular mechanisms that promote odontogenic differentiation of hDPSCs are essential for further investigate. 
Long non-coding RNAs (IncRNAs) play crucial roles in numerous physiological and pathological activities in cells. A variety of functions of IncRNAs were found in recent years in embryonic development, inflammation, cell differentiation and tumors ${ }^{[4-6]}$. Recent studies have shown that IncRNAs can regulate the differentiation of stem cells ${ }^{[7-9]}$. Several functions of IncRNAs in biological mineralization have been elucidated ${ }^{[10]}$. However, few studies have focused on the role of IncRNAs and their molecular mechanisms underlying odontoblastic differentiation of hDPSCs.

In this study, IncRNA microarray results revealed that IncRNA-H19 $(\mathrm{H} 19)$ was significantly up-regulated in hDPSCs undergoing odontoblastic differentiation. We investigated the function role of H19 in the odontoblastic differentiation of hDPSCs. Overexpression of $\mathrm{H} 19$ notably promoted odontogenic differentiation of hDPSCs in vitro and in vivo. In accordance with the bioinformatics analysis, the mineralization-related miR-140-5p which had potential binding sites with $\mathrm{H} 19$ was selected for further study. We demonstrated that $\mathrm{H} 19$ could accelerate odontogenic differentiation of hDPSCs via binding miR-140-5p to up-regulate bone morphogenetic protein-2(BMP-2) and fibroblast growth factor 9(FGF9) expression. Therefore, this research may thus provide a novel regulatory mechanism of IncRNAs in odontogenic differentiation.

\section{Materials And Methods}

\section{Cell culture}

All DPSCs in our experiments derived from human patients. Cells were isolated from the extracted third molars of human dental patients between 18 and 22 years old. The process were reviewed and approved by the institutional Review Board of the Hospital of Stomatology of Guangzhou Medical University, and a signed informed consent document was obtained from each patient. hDPSCs were prepared using explants and the trypsin digestion technique. Cells were cultured and expanded in alpha minimum essential medium (a-MEM) supplemented with 10\% (v/v) fetal bovine serum (FBS) and 1\% (v/v) penicillin-streptomycin solution. Cells were maintained in a humidified atmosphere of $5 \% \mathrm{CO}_{2}$ at $37{ }^{\circ} \mathrm{C}$. Fresh culture media was replenished every 3 days, Third-passage cells were harvested for the subsequent experiments.

\section{Flow cytometry assay}

Cultured cells in their 3rd passage were identified based on the surface antigens of hDPSCs using a flow cytometry method. Cells were trypsinized and incubated in PBS containing $0.1 \%$ FBS for 45 min with fluorescein-conjugated monoclonal antibodies against CD34, CD45, CD73, CD90, CD146 and CD166 (BD Biosciences, San Jose, CA, USA). Flow cytometry analysis was performed using a flow cytometer (FACSCalibur, BD Biosciences). 


\section{LncRNA microarray}

After odotoblastic induction for 3 days, the differentiated hDPSCs in the culture dishes were isolated and hDPSCs cultured in normal culture medium were observed as the control. Total RNA was isolated with TRIzol (Invitrogen) according to the manufacturer's instructions. A LncRNA microarray (KangChen Biotech, Shanghai, China) using induced group and non-induced group was performed. Differentially expressed IncRNAs were identified based on fold change $(>2)$, as well as $P<0.05$.

\section{Odontogenic differentiation induction and treatment of hDPSCs}

hDPSCs (passages 4 6) were seeded in 6-well plates at a density of $1 \times 10^{5}$ cells each well. To induce odontogenic differentiation of hDPSCs, cells were cultured with odontogenic medium (10\% FBS, $10^{-8}$ $\mathrm{mol} / \mathrm{L}$ dexamethasone, $10 \mathrm{mmol} / \mathrm{L} \beta$-glycerophosphate and $50 \mathrm{mg} / \mathrm{mL}$ ascorbic acid in a-MEM) for 14 days. Control samples were cultured in $10 \%$ FBS in a-MEM with no supplements. For functional experiments, recombinant human protein BMP-2 (rhBMP-2; Gibco, USA) and FGF9 (rhFGF9; Peprotect, USA) were purchased and used to stimulate hDPSCs. hDPSCs were cultured with odontogenic medium consisting of rhBMP-2 $(20 \mathrm{ng} / \mathrm{mL})$ and rhFGF9 $(20 \mathrm{ng} / \mathrm{mL})$ separately. Fresh medium was changed every 3 days.

\section{ALP assay and Alizarin Red S staining}

Cells lysates were harvested after odontoblatic differentiation, and ALP activity was evaluated and analyzed following the manufacturer's instructions (ALP Diagnostics Kit, Yeasen, Shanghai, China). hDPSCs were fixed in $4 \%$ paraformaldehyde, and mineral nodules were detected by Alizarin Red $S$ staining (BestBio, Shanghai, China).

\section{RNA preparation and qRT-PCR}

Total RNA was isolated from cultured cells with TRIzol Reagent (Invitrogen, USA) according to the manufacturer's protocol. Reverse transcription was performed with PrimeScript RT Master Mix (TaKaRa, Japan) for IncRNA and mRNA. For miRNA examination, cDNA was synthesized using a miRNA First Strand cDNA Synthesis Kit (Sangon Biotech, China). RNA expression was measured by quantitative realtime PCR analysis using a CFX96 Real-Time PCR instrument (Life Technology, USA) and SYBR Green reagent (TaKaRa, Japan). GAPDH was used as an internal control for IncRNA and mRNA. The expression of miRNA was normalized to U6. Primers were synthesized by Generay Technologies (Shanghai, China), and all sequences are available in Additional file 1: Table 1. The $2^{-\Delta \Delta C T}$ method was used to calculate relative expression levels. 


\section{Western blot analysis}

Cells were lysed in RIPA Buffer (Beyotime, China) combined with a cocktail of protease inhibitors (Thermo Scientific, Rockford, IL). Equal amounts of proteins $(25 \mu \mathrm{g})$ of different groups were separated by $10 \%$ SDS-PAGE and transferred to 0.45- $\mu \mathrm{m}$ PVDF membranes (Millipore, USA). The membrane was first blocked with $5 \%$ BSA for $1 \mathrm{~h}$ at room temperature and incubated at $4{ }^{\circ} \mathrm{C}$ overnight with primary antibodies: GAPDH (1:1000; Abcam, Cambridge, UK), RUNX2(1:1000; Cell Signaling Technology, Danvers, MA), DSPP (1:1000; Abcam, Cambridge, UK) and DMP-1 (1:1000; Abcam, Cambridge, UK). After washed with Tris-buffer saline containing $0.05 \%$ Tween 20 (TBST) for three times and 5 min each, the membranes were incubated with secondary antibodies labelled with horseradish peroxidase (1:3000; Cell Signaling Technology, Danvers, MA) at room temperature for $1 \mathrm{~h}$. Blots were visualized using ECL Western Blotting Substrate.

\section{Cell transfection}

Recombinant lentiviruses targeting $\mathrm{H} 19$ (shH19-1 and shH19-2) and Lenti-shNC were purchased from GenePharma Company (Shanghai, China). hDPSCs were transfected by lentiviruses exposure in $1 \mathrm{~mL}$ aMEM supplemented with $10 \%$ FBS and $5 \mu \mathrm{g} / \mathrm{mL}$ polybrene for $24 \mathrm{~h}$. H19 overexpression plasmid pcDNA3.1-H19, miR-140-5p mimics and and scramble control (NC) were chemically synthesized by GenePharma. When hDPSCs were $70 \%-80 \%$ confluent, pcDNA-H19 and miRNA mimic transfection was performed using Lipofectamine 3000 (Invitrogen, USA) according to the manufacturer's instructions. qRTPCR analysis was used to detect $\mathrm{H} 19$ and miR-140-5p expression levels to validate the transfection efficiencies. The cells were cultured in mineralizing medium for odontoblastic differentiation $48 \mathrm{~h}$ after transfection.

\section{Dual luciferase reporter assay}

The luciferase reporter vector psiCHECK-2 containing full-length of H19 sequence, the 3' untranslated region (UTR) sequence of BMP-2/FGF9 and their relevant mutant types were synthesized by Synbio, China. HEK293T cells were seeded into 24 -well plates at a density of $5 \times 10^{4}$ cells per well the day before transfection. Cells were transiently co-transfected with corresponding psiCHECK-2 vector and miRNC/miR-140-5p mimics using Lipofectamine 3000. Renilla and Firefly luciferase activity were measured separately $48 \mathrm{~h}$ after transfection using a dual-luciferase reporter assay system (Promega, USA) following the manufacturer's instructions. These experiments were repeated three times. 


\section{ELISA assay}

The Human Enzyme-linked immunosorbent assay (ELISA) kits were obtained from CLOUD-CLONE CORP (Wuhan, China). The culture supernatants were collected on day 2 after odontogenic induction treatment. The secreted levels of BMP-2 and FGF9 were quantified using corresponding ELISA kits, according to the manufacturer's protocol.

\section{In vivo odontogenesis assay}

$\mathrm{BALB} / \mathrm{c}$ nude mice (5 weeks old, five mice were included in each group) were purchased from the Experimental Animal Center, Guangzhou University of Chinese Medicine. All the animal care and experimental procedures were approved by the Institutional Animal Care and Use Committee of Guangzhou University of Chinese Medicine and were performed in accordance with established guidelines. hDPSCs with $\mathrm{H} 19$ overexpression and negative controls induced under odontoblastic medium for 1 week were harvested and incubated with Bio-Oss Collagen (Geistlich, Germany) scaffolds for $1 \mathrm{~h}$ at $37^{\circ} \mathrm{C}$. The hDPSCs-loaded scaffolds were implanted subcutaneously into the nude mice. Implants were harvested 6 weeks after implantation and fixed in 4\% PFA.

\section{Analyses of bone formation in vivo}

Micro-CT analysis was performed using a high-resolution Micro-CT (Bruker, Karlsruhe, Germany) set as $10 \mu \mathrm{m}$ of the voxel resolution of the scanned volumes, $500 \mathrm{~ms}$ of the 360 rotational steps per time. MicroCT image slices were reconstructed and the ratio of new bone volume to existing tissue volume (BV/TV) were calculated by Micro-CT image analysis software. For histological examination, the specimens were decalcified in 10\% EDTA ( $\mathrm{pH}$ 7.4) for 30 days. After dehydrated and embedded with paraffin, tissue samples of $5 \mu \mathrm{m}$ thick paraffin section were stained with hematoxylin and eosin (H\&E) and Masson's trichrome. Immunohistochemical staining was conducted using HRP/DAB (ABC) detection IHC kit and human-specific primary antibodies, including DMP-1 (1:100). All products for IHC were from Abcam. Histological staining was captured under the microscope (Leica Microsystems, Germany) with an increase of $\times 100$.

\section{Statistical analysis}

Statistical analyses were performed using GraphPad Prism7.0 (GraphPad Prism, Inc., La Jolla, CA, USA). One-way analysis of variance (ANOVA) and Student's t test (two-tailed) were used to evaluate the statistical significance. All data are shown as the means \pm SD from three independent experiments. Statistical significance was defined as $P<0.05$. 


\section{Results}

\section{Characteristics of hDPSCs derived from adult dental pulp}

hDPSCs were identified with flow cytometry. hDPSCs exhibited high expression of CD73 (90.7\%), CD90 (100\%), CD146 (10.6\%), and CD166 (12.9\%) and were negative for CD34 (0.1\%) and CD45 (0.1\%) (Fig. 1AB). These results indicated that hDPSCs highly expressed mesenchymal cell surface molecular markers and scarcely expressed hematopoietic system-derived cell surface markers. Furthermore, the differentiation capacities of hDPSCs were assessed. The mRNA expression levels of odontogenesisrelated genes DSPP and DMP-1 were up-regulated gradually during odontogenic differentiation (Fig. 1C). Western blot analysis revealed a similar trend that the protein levels of DSPP and DMP-1 were also enhanced significantly after odontogenic induction (Fig. 1D). Matrix mineralization and ALP activity were increased significantly in the process of odontogenic induction as compared to the normal culture group (Fig. 1E-F).

\section{Microarray expression profile analysis of IncRNAs in hDPSCs during differentiation induction}

Whether IncRNAs involved in the odontoblastic differentiation of hDPSCs were verified by microarray. Compared with the normal culture group, 1106 IncRNAs were identified to significantly differentially expressed (fold change $>2.0 ; \mathrm{P}<0.05$ ) after 3 days of odontoblastic induction in hDPSCs. Among these, 617 IncRNAs were up-regulated, while 489 IncRNAs were down-regulated (Additional file 1: Figure S1A). Among the significantly up-regulated IncRNAs, mineralization-related H19, MALAT1, MIR31HG and WNT2 were chosen as candidate IncRNAs. To prove the accuracy of the microarray results, qRT-PCR was used to investigate the expression level of four IncRNAs at each time point during differentiation induction. It revealed that $\mathrm{H} 19$ was significantly up-regulated 5.9-fold after induction for 7 days (Additional file 1: Figure S1B-E). Therefore, we focused on H19 for further study.

\section{H19 promotes the odontoblastic differentiation of hDPSCs}

To investigate the function of $\mathrm{H} 19$, we stably silenced $\mathrm{H} 19$ expression in hDPSCs with lentiviruses. The transfection effects were observed under an inverted fluorescence microscope. Enhanced Green Fluorescent Protein (EGFP) showed that hDPSCs were infected with the lentiviruses (Fig. 2A). qRT-PCR indicated that the expression level of $\mathrm{H} 19$ was down-regulated by approximately $74.3 \%$ in shH19-1 group and $79.3 \%$ in shH19-2 group compared with that of the sh-NC group ( $\mathrm{P}<0.01$ vs control group) (Fig. 2B). After odontoblastic induction for 14 days, down-regulation of $\mathrm{H} 19$ resulted in significantly inhibited odontoblastic differentiation, characterized by lower expression levels of DSPP and DMP-1, weaker ALP activity and fewer mineralization nodules (Fig. 2C-F). Correspondingly, the expression level of $\mathrm{H} 19$ was increased approximately 13 -fold after transfected with overexpression plasmid (Fig. 2G). Consistent with 
the results above, the forced expression of $\mathrm{H} 19$ led to a stronger hDPSC capacity to differentiate into odontoblasts during odontoblastic induction for 14 days (Fig. 2H-K).

\section{$\mathrm{H} 19$ facilitates the odontogenesis of hDPSCs in vivo}

To further confirm the role of $\mathrm{H} 19$ in odontoblastic differentiation of hDPSCs, the in vivo experiments were conducted. After induction culture for 1 week in vitro, the $\mathrm{H} 19$ overexpression and negative control transfected hDPSCs were loaded on Bio-Oss Collagen scaffolds. The seeded scaffolds were then gently implanted subcutaneously into BALB/c nude mice (five mice per group) for 6 weeks (Fig. 3A). Threedimensional reconstructed micro-CT analysis revealed the BV/TV increased significantly in H19overexpressing group compared with the control group (Fig. 3B). The histological observations revealed a similar trend of BV/TV. H\&E staining indicated more dentin-like structures formation around the area of scaffolds in H19-overexpressing group. In addition, more blue-colored bone collagen deposit was observed by Masson staining in H19-overexpression group, compared with the control group. Moreover, IHC analysis was applied to detect the protein expression of DMP-1 in specimens of each group. The abundance of DMP-1 was increased obviously in H19-overexpressing group, suggesting that upregulation of H19 could promote odontogenic differentiation of hDPSCs in vivo (Fig. 3C).

\section{H19 serves as a miRNA sponge for miR-140-5p in hDPSCs}

Computational analysis was performed using Starbase (http://starbase.sysu.edu.cn), DIANA-LncBase (http://www.microrna.gr/LncBase) and RegRNA (http://regrna2.mbc.nctu.edu.tw) databases, and six collectively predicted miRNAs (miR-17-5p, miR-93-5p, miR-103a-3p, miR-106b-5p, miR-140-5p and miR148-5p) may act as biological targets of H19 (Fig. 4A). The expression patterns of the candidate miRNAs in hDPSCs during odontoblastic differentiation were detected by qRT-PCR. Impressively, the expression levels of miR-140-5p were significantly decreased compared with those of the non-induction group (Fig. 4B). In addition, we conducted a dual-luciferase reporter assay to validate the interaction between $\mathrm{H} 19$ and miRNAs. The results revealed that miR-140-5p significantly reduced the luciferase activity of H19wild compared to that of H19-mut, indicating that H19 might function as a sponge of miR-140-5p (Fig. 4C-D).

\section{Overexpression of miR-140-5p inhibits odontoblastic differentiation}

To investigate whether miR-140-5p play a role in regulating odontoblastic differentiation, miR-140-5p mimics were transfected into hDPSCs and the efficiency was evaluated $48 \mathrm{~h}$ after transfection. The qRTPCR results showed that miR-140-5p transfected hDPSCs had an 8.9-fold higher miR-140-5p expression compared with miR-NC mimics transfected hDPSCs (Fig. 4E). Following odontoblastic induction for 7 
and 14 days, relative mRNA expressions of ALP, RUNX2, DSPP and DMP-1 were markedly suppressed in the miR-140-5p overexpression group as compared to the mimics NC group (Fig. 4F-G). Consistently, western blot assay revealed that the odontogenic markers RUNX2, DSPP and DMP-1 were greatly decreased in miR-140-5p overexpression group (Fig. 4H). In addition, after odontoblastic induction for 7 days, the matrix mineralization level was remarkably decreased in miR-140-5p overexpression group assessed by Alizarin Red S staining. Overexpression of miR-140-5p continued to decrease calcified nodules after induction culture for 14 days (Fig. 4I). Briefly, these results indicated that miR-140-5p could suppress the odontoblastic differentiation of hDPSCs.

\section{H19 regulates BMP-2/FGF9 expression by binding to miR-140-5p}

Further prediction of target genes of miR-140-5p was performed by miRDB (http://mirdb.org) and TargetScan (http://www.targetscan.org/vert_72/). Notably, we found that the 3'untranslated region (UTR) of the mineral-associated genes BMP-2 and FGF9 contained miR-140-5p binding sites (Fig. 5A). To confirm this finding, luciferase reporter assays were performed using psiCHECK-2 vector containing wildtype or mutant version of BMP-2/FGF9 3'untranslated region (3'UTR) respectively. After co-transfected with miR-140-5p mimics and psiCHECK-2 vector for $48 \mathrm{~h}$ in HEK293T cells, the luciferase reporter results showed that obviously reduced luciferase activity was observed in BMP-2-wild not in BMP-2-mut. Meanwhile, the luciferase activity was remarkably reduced in HEK293T cells co-transfected with miR-1405p mimics and FGF9-wt 3'UTR vector, but not significantly changed in HEK293T cells co-transfected with miR-140-5p mimics and the FGF9-mut 3'UTR vector (Fig. 5B). Furthermore, we conducted rescue assays to estimate whether BMP-2 and FGF9 act as downstream targets of H19/miR-140-5p by co-transfecting $\mathrm{H} 19$ and miR-140-5p mimics in hDPSCs. The RT-PCR analysis and ELISA assay results showed that transfection with miR-140-5p mimics inhibited the expression of BMP-2 and FGF9 in hDPSCs, while cotransfected with $\mathrm{H} 19$ could partly abolished this effect. The above findings confirmed BMP-2 and FGF9 are direct targets of miR-140-5p (Fig. 5C-D).

After elucidated that BMP-2 and FGF9 are direct targets of miR-140-5p, we next gain further insights into the biological influence of BMP-2 and FGF9 on hDPSCs. The ELISA assay results revealed the expressions of BMP-2 and FGF9 increased along with odontogenesis process (Fig. 5E). Since BMP-2 and FGF9 were secretory proteins, exogenous BMP-2 and FGF9 were utilized to stimulate hDPSCs. Odontogenic induction medium with $20 \mathrm{ng} / \mathrm{mL}$ recombinant BMP-2 or FGF9 were treated with hDPSCs separately for 14 days. Subsequent qRT-PCR assay and western blot assay revealed that exogenous BMP-2/FGF9 facilitated the gene expression levels of odontogenic markers, ALP, Runx2, DSPP, and DMP1, compared with the common odontogenic induction group (Fig. 5F-G). Meanwhile, the Alizarin Red S staining indicated that odontoblastic differentiation ability of hDPSCs treated with BMP-2/FGF9 was enhanced (Fig. $5 \mathrm{H}$ ). Taken together, these data indicated that H19 acts upstream of miR-140-5p and inhibits the effect of miR-140-5p to target BMP-2/FGF9. 


\section{H19 abrogates the inhibitory effect of miR-140-5p on odontoblastic differentiation}

Since $\mathrm{H} 19$ plays a ceRNA role by binding to miR-140-5p, rescue experiments were performed to validate whether miR-140-5p was involved in H19-mediated odontoblastic differentiation in hDPSCs. For the rescue assays, the $\mathrm{H} 19$ overexpression plasmid and miR-140-5p mimics were co-transfected into hDPSCs. The down-regulated expression of odontoblast-related genes indicated that miR-140-5p mimics attenuated the promoting odontoblastic differentiation effect of H19 (Fig. 6A). Western blot assays revealed that the effects of miR-140-5p inhibition on the odontoblastic differentiation of hDPSCs could be significantly reversed by overexpressing H19 (Fig. 6B). Moreover, Alizarin Red S staining revealed that the overexpression of miR-140-5p overcame the promotion effects of increased H19 on odontoblastic differentiation (Fig. 6C). A summarized figure revealed the role and regulatory mechanisms of H19 in this study (Fig. 6D).

\section{Discussion}

Human Dental pulp stem cells (hDPSCs) was first isolated and named by Gronthos ${ }^{[11]}$. The odontoblastic differentiation of hDPSCs is a crucial factor in reparative dentin generation and dental tissue self-repair in inflammatory microenvironments ${ }^{[12]}$.

LncRNAs are involved in a variety of physiological and pathological activities of cells, including proliferation, differentiation, migration and apoptosis ${ }^{[13-15]}$. Previous studies have revealed that IncRNAs play important roles in osteogenic differentiation of mesenchymal stem cells (MSCs) ${ }^{[16-19]}$. In this study, IncRNA microarray analysis using differentiation-induced and un-induced hDPSCs was performed to determine whether IncRNAs implicated in the odontoblastic differentiation of hDPCs. According to the microarray expression profiles, the expression levels of 1106 IncRNAs were significantly changed by more than 2.0-fold; 617 of these were up-regulated, and 489 were down-regulated. Among those IncRNAs, MALAT1, MIR31HG, H19 and WNT2 were selected for further identification, based on reports that concentrated on IncRNAs regulating biological mineralization ${ }^{[20,21]}$. qRT-PCR results were most consistent with the IncRNA microarray results that $\mathrm{H} 19$ expression increased remarkably at day 7 after mineralization induction, so $\mathrm{H} 19$ was chosen as a candidate IncRNA.

We subsequently evaluated the functional effects of $\mathrm{H} 19$ by transfecting lentiviruses or plasmids into hDPSCs. Overexpression of H19 significantly promoted the odontoblastic differentiation of hDPSCs, whereas lentiviruses-mediated silencing of $\mathrm{H} 19$ had the opposite effects on hDPSCs. In addition, an ectopic odontogenesis nude mice model was used to further confirm the role of H19 in hDPSCs in vivo. Both micro-CT analysis and histological examination revealed significantly increased levels of dentin-like structures formation and greater abundance of odontogenic specific markers DMP-1 in H19overexpression hDPSCs loaded group, which were consistent with the in vitro experiments. Our results were consistent with Zeng $\mathrm{L}$ et al. ${ }^{[29]} \mathrm{H} 19$ plays a critical role in the differentiation of many vital organs and plays pivotal roles in the differentiation of mesenchymal stem cells ${ }^{[22-25]}$. A. Keniry et al. ${ }^{[26]}$ found that $\mathrm{H} 19$ can enhance the expression level of RUNX2 to promote bone regeneration. Zhou et al. [27] 
revealed that $\mathrm{H} 19$ can help in bone tissue reparation by inhibiting p53. As a biomineralization promoter, $\mathrm{H} 19$ can promote the osteogenic differentiation of mesenchymal stem cells (MSCs) through the Notch signaling pathway ${ }^{[24]}$. One recent study revealed that $\mathrm{H} 19$ enhanced the osteo/dentinogenesis of SCAPs in vivo via the miR-141/SPAG9 pathway ${ }^{[28]}$. However, the regulatory effect of $\mathrm{H} 19$ in odontoblastic differentiation and underlying molecular mechanisms were not entirely identified.

Numerous studies have demonstrated that IncRNA mainly act as miRNA sponges to play various regulatory roles ${ }^{[30-32]}$. Here, we hypothesized that $\mathrm{H} 19$ could promote the odontoblastic differentiation of hDPSCs via sponging miRNAs. Three database, including Starbase, DIANA-LncBase and RegRNAD predicted six mineralization-related miRNAs (miR-17-5p, miR-93-5p, miR-103a-3p, miR-106b-5p, miR-140$5 p$ and miR-148-5p) that may have potential binding sites with H19. The expression levels of these candidate miRNAs were evaluated after 14 days of induction. Compared with the non-induction group, the expression levels of miR-140-5p were remarkably decreased in the induction group. Dual-luciferase assay was performed to affirm the direct binding between $\mathrm{H} 19$ and miR-140-5p. Later results confirmed that the inhibitory effect of miR-140-5p on odontoblastic differentiation by transfecting miRNA mimics into hDPSCs. The expression levels of odontoblast-related genes and matrix mineralization levels were obviously decreased by overexpression of miR-140-5p, compared with miR-NC mimics.

Zheng $L$ et. al. ${ }^{[33]}$ revealed that IncRNA MEG3 promoted the osteogenesis of hADSCs via sponging miR$140-5 p$, indicating the inhibitory role of miR-140-5p in osteogenic differentiation. Our rescue experiments indicated that $\mathrm{H} 19$ could partially abrogate the inhibitory effects on odontoblastic differentiation induced by miR-140-5p in vitro which implied that $\mathrm{H} 19$ is involved in the ceRNA regulatory network and acts as a miR-140-5p sponge. This is the first report about the regulation of H19/miR-140-5p axis in odontogenic differentiation of hDPSCs. These results showed that $\mathrm{H} 19$ promoted the odontoblastic differentiation of hDPSCs by interacting with miR-140-5p. Our findings provided an explanation for the mechanism role of $\mathrm{H} 19$ in odontogenesis.

Lu X et. al. ${ }^{[34]}$ reported that miR-140-5p regulated the odontoblastic differentiation of DPSCs via targeting Wnt1/ $\beta$-catenin signaling. Sun $D$ et. al. ${ }^{[35]}$ found that miR-140-5p-mediated regulation of the proliferation and differentiation of human dental pulp stem cells occurs through the lipopolysaccharide/toll-like receptor 4 signaling pathway. However, the downstream target genes regulated by H19/mir-140-5p axis remains unclear. To reveal the downstream molecular mechanism of H19/miR-140-5p pathway regulating the odontoblastic differentiation of hDPSCs, miRDB and Targetscan were applied to search for potential targets. BMP-2 and FGF9 were predicted as the candidate target genes since the 3 '-UTR region of BMP-2 and FGF9 contained potential binding sites with miR-140-5p respectively. Dual-luciferase reporter assays were conducted to further confirm the interaction. Overexpression of miR-140-5p significantly reduced the luciferase activity in BMP-2-wild and FGF9-wild groups, compared with HEK293T cells co-transfected with miR-140-5p mimics and the BMP-2-mut/FGF9-mut 3'UTR vector groups separately. Hwang ${ }^{[39]}$ found that miR-140-5p suppressed BMP2-mediated osteogenesis in undifferentiated human mesenchymal stem cells. Rothman AM et al. ${ }^{[40]}$ revealed that miR-140-5p mimic 
altered BMP signaling in pulmonary arterial smooth muscle cells. Much of the literature acknowledges that FGF9 is essential for osteogenesis ${ }^{[41-43]}$,but the relationship between FGF9 and H19 has not been described. Overexpression of $\mathrm{H} 19$ increased the expression level of BMP-2 and FGF9, whereas overexpression of miR-140-5p decreased BMP-2/FGF9 mRNA and protein expression. The expression level of BMP-2/FGF9 were partly increased in hDPSCs co-transfected with $\mathrm{H} 19$ and miR-140-5p mimics compared to hDPSCs transfected with miR-140-5p mimics alone. Therefore, our results indicated that H19 regulated BMP-2/FGF9 expression by inhibiting the effect of miR-140-5p.

In this study, the biological role of BMP-2 and FGF9 in regulating the odontogenic differentiation of hDPSCs have been verified. hDPSCs cultured in osteogenic induction medium enriched with BMP-2 or FGF9 separately, were demonstrated to encourage osteogenic differentiation compared to the normal osteogenic induction group. Furthermore, the results showed that the odontogenic capacity of BMP-2 was better than that of FGF9. It is well recognized that BMP-2 is a critical growth factor and important biomarker involved in osteo/odontoblastic differentiation and bone formation ${ }^{[36-38]}$. Our results revealed the similar trend that BMP-2 offered a strong signal for differentiation and mineralization of hDPSCs, but was different from Lu $\mathrm{J}$ et $\mathrm{a}^{[44]}$ which indicated that exogenous FGF9 suppress osteogenic differentiation. This difference may be due to the different stage of hDPSCs and the compensation effect between BMP-2 and FGF9.

In summary, our results indicated that $\mathrm{H} 19 / \mathrm{miR}-140-5 \mathrm{p}$ pathway promoted the odontoblastic differentiation of hDPSCs partially through the regulation of BMP-2 and FGF9.

\section{Conclusion}

In summary, our results demonstrated that $\mathrm{H} 19$ was up-regulated in hDPSCs during odontogenic induction. Mechanistically, H19 could significantly promote odontogenic differentiation of hDPSCs through competitively binding to miR-140-5p and subsequently reduce the inhibitory effects of miR-140$5 p$ on BMP-2 and FGF9.

\section{List Of Abbreviations}

hDPSCs: human dental pulp stem cells; IncRNA: long non-coding RNA; cDNA: complementary DNA; qRTPCR: quantitative real-time polymerase chain reaction; DSPP: dentin sialophosphoprotein; DMP-1: dental matrix protein-1; ALP: alkaline phosphatase; RUNX2: runt-related transcription factor 2; BMP-2: Bone Morphogenetic Protein 2; FGF9: fibroblast growth factor 9; hBMSCs: human bone mesenchymal stem cells; MSCs: mesenchymal stem cells; ceRNA: competing endogenous RNA; miRNA: microRNA; 3'UTR: 3' untranslated region; NC: negative control.

\section{Declarations}


All experiments using cells derived from human patients were reviewed and approved by the institutional Review Board of the Hospital of Stomatology of Guangzhou Medical University. All the animal care and experimental procedures were approved by the Institutional Animal Care and Use Committee of Guangzhou University of Chinese Medicine and were performed in accordance with established guidelines.

\section{CONSENT FOR PUBLICATION}

Written informed consent for publication was obtained from all participants.

\section{AVAILABILITY OF DATA AND MATERIALS}

The datasets used or analysed during the current study are available from the corresponding author on reasonable request.

\section{CONFLICT OF INTERESTS}

The authors declare that they have no conflict of interest.

\section{FUNDING}

This work was supported by the Science and Technology Program of Guangdong Provincial Science and Technology Department (No.2016ZC0134), Guangdong Provincial Science and Technology Innovation Strategy Special Fund, and Guangdong-Hong Kong Joint Funding Program (platform) Project (No.2018B050502012).

\section{AUTHORS' CONTRIBUTIONS}

QZJ and YGJ conceived the study. JLZ and XRT designed the research and performed the experiments. YYK, LYG. and WCZ. analyzed the data. BSL and YC performed statistical analysis. JLZ, XRT and QZJ wrote and reviewed the manuscript. All authors read and approved the final manuscript.

\section{ACKNOWLEDGEMENTS}


Not applicable.

\section{References}

1. Kunimatsu R, Nakajima K, Awada T, Tsuka Y, Abe T, Ando K, Hiraki T, Kimura A, Tanimoto K: Comparative characterization of stem cells from human exfoliated deciduous teeth, dental pulp, and bone marrow-derived mesenchymal stem cells. Biochem Bioph Res Co 2018, 501(1):193-198.

2. Yu F, Dong Y, Yang YW, Lin PT, Yu HH, Sun X, Sun XF, Zhou H, Huang L, Chen JH: Effect of an Experimental Direct Pulp-capping Material on the Properties and Osteogenic Differentiation of Human Dental Pulp Stem Cells. Sci Rep 2016, 6:34713.

3. Leyendecker A, Pinheiro CCG, Fernandes TL, Bueno DF: The use of human dental pulp stem cells for in vivo bone tissue engineering: A systematic review. Journal of Tissue Engineering 2018, 9.

4. Chen X, He L, Zhao Y, Li Y, Zhang S, Sun K, So K, Chen F, Zhou L, Lu L et al: Malat1 regulates myogenic differentiation and muscle regeneration through modulating MyoD transcriptional activity. Cell Discov 2017, 3:17002.

5. Huang Y, Jin C, Zheng Y, Li X, Zhang S, Zhang Y, Jia L, Li W: Knockdown of IncRNA MIR31HG inhibits adipocyte differentiation of human adipose-derived stem cells via histone modification of FABP4. Sci Rep 2017, 7(1):8080.

6. Wang $Y$, Yao J, Meng H, Yu Z, Wang Z, Yuan X, Chen H, Wang A: A novel long non-coding RNA, hypoxia-inducible factor-2alpha promoter upstream transcript, functions as an inhibitor of osteosarcoma stem cells in vitro. Mol Med Rep 2015, 11(4):2534-2540.

7. Chen MT, Lin HS, Shen C, Ma YN, Wang F, Zhao HL, Yu J, Zhang JW: PU.1-Regulated Long Noncoding RNA Inc-MC Controls Human Monocyte/Macrophage Differentiation through Interaction with MicroRNA 199a-5p. Mol Cell Biol 2015, 35(18):3212-3224.

8. Lee S, Seo HH, Lee CY, Lee J, Shin S, Kim SW, Lim S, Hwang KC: Human Long Noncoding RNA Regulation of Stem Cell Potency and Differentiation. Stem Cells Int 2017, 2017:6374504.

9. Zhang W, Dong R, Diao S, Du J, Fan Z, Wang F: Differential long noncoding RNA/mRNA expression profiling and functional network analysis during osteogenic differentiation of human bone marrow mesenchymal stem cells. Stem Cell Res Ther 2017, 8(1):30.

10. Park KS, Mitra A, Rahat B, Kim K, Pfeifer K: Loss of imprinting mutations define both distinct and overlapping roles for misexpression of IGF2 and of H19 IncRNA. Nucleic Acids Res 2017, 45(22):12766-12779.

11. Gronthos S, Mankani M, Brahim J, Robey PG, Shi S: Postnatal human dental pulp stem cells (DPSCs) in vitro and in vivo. Proc Natl Acad Sci U S A 2000, 97(25):13625-13630.

12. Cooper PR, Takahashi Y, Graham LW, Simon S, Imazato S, Smith AJ: Inflammation-regeneration interplay in the dentine-pulp complex. J Dent 2010, 38(9):687-697.

13. Hu X, Duan L, Liu H, Zhang L: Long noncoding RNA LINC01296 induces non-small cell lung cancer growth and progression through sponging miR-5095. Am J Trans/ Res 2019, 11(2):895-903. 
14. Liu M, Li B, Peng W, Ma Y, Huang Y, Lan X, Lei C, Qi X, Liu GE, Chen H: LncRNA-MEG3 promotes bovine myoblast differentiation by sponging miR-135. J Cell Physio/2019.

15. Yu G, Wang W, Deng J, Dong S: LncRNA AWPPH promotes the proliferation, migration and invasion of ovarian carcinoma cells via activation of the Wnt/betacatenin signaling pathway. Mol Med Rep 2019.

16. Jia B, Wang Z, Sun X, Chen J, Zhao J, Qiu X: Long noncoding RNA LINC00707 sponges miR-370-3p to promote osteogenesis of human bone marrow-derived mesenchymal stem cells through upregulating WNT2B. Stem Cell Res Ther 2019, 10(1):67.

17. Sun $H$, Peng $G$, Ning $X$, Wang J, Yang $H$, Deng J: Emerging roles of long noncoding RNA in chondrogenesis, osteogenesis, and osteoarthritis. Am J Transl Res 2019, 11(1):16-30.

18. Wang CG, Liao Z, Xiao H, Liu H, Hu YH, Liao QD, Zhong D: LncRNA KCNQ10T1 promoted BMP2 expression to regulate osteogenic differentiation by sponging miRNA-214. Exp Mol Pathol 2019, 107:77-84.

19. Peng S, Cao L, He S, Zhong Y, Ma H, Zhang Y, Shuai C: An Overview of Long Noncoding RNAs Involved in Bone Regeneration from Mesenchymal Stem Cells. Stem Cells Int 2018, 2018:8273648.

20. Yang Q, Jia L, Li X, Guo R, Huang Y, Zheng Y, Li W: Long Noncoding RNAs: New Players in the Osteogenic Differentiation of Bone Marrow- and Adipose-Derived Mesenchymal Stem Cells. Stem Cell Rev 2018, 14(3):297-308.

21. Fan JB, Zhang Y, Liu W, Zhu XH, Xu DW, Zhao JN, Cui ZM: Long Non-Coding RNA MALAT1 Protects Human Osteoblasts from Dexamethasone-Induced Injury via Activation of PPM1E-AMPK Signaling. Cell Physiol Biochem 2018, 51(1):31-45.

22. Ma L, Tian X, Wang F, Zhang Z, Du C, Xie X, Kornmann M, Yang Y: The long noncoding RNA H19 promotes cell proliferation via E2F-1 in pancreatic ductal adenocarcinoma. Cancer Biol Ther 2016, 17(10):1051-1061.

23. Li G, Yun X, Ye K, Zhao H, An J, Zhang X, Han X, Li Y, Wang S: Long non-coding RNA-H19 stimulates osteogenic differentiation of bone marrow mesenchymal stem cells via the microRNA-149/SDF-1 axis. J Cell Mol Med 2020.

24. Liao J, Yu X, Hu X, Fan J, Wang J, Zhang Z, Zhao C, Zeng Z, Shu Y, Zhang R et al: IncRNA H19 mediates BMP9-induced osteogenic differentiation of mesenchymal stem cells (MSCs) through Notch signaling. Oncotarget 2017, 8(32):53581-53601.

25. Xiaoling G, Shuaibin L, Kailu L: MicroRNA-19b-3p promotes cell proliferation and osteogenic differentiation of BMSCs by interacting with IncRNA H19. BMC Med Genet 2020, 21(1):11.

26. Keniry A, Oxley D, Monnier P, Kyba M, Dandolo L, Smits G, Reik W: The H19 lincRNA is a developmental reservoir of miR-675 that suppresses growth and Igf1r. Nat Cell Biol 2012, 14(7):659665.

27. Zhou QP, Zhang F, Zhang J, Ma D: H19 promotes the proliferation of osteocytes by inhibiting p53 during fracture healing. Eur Rev Med Pharmacol Sci 2018, 22(8):2226-2232. 
28. Li ZH, Yan M, Yu Y, Wang YQ, Lei G, Pan Y, Li N, Gobin R, Yu JH: LncRNA H19 promotes the committed differentiation of stem cells from apical papilla via miR-141/SPAG9 pathway. Cell Death \& Disease 2019, 10.

29. Zeng L, Sun S, Han D, Liu Y, Liu H, Feng H, Wang Y: Long non-coding RNA H19/SAHH axis epigenetically regulates odontogenic differentiation of human dental pulp stem cells. Cell Signal 2018, 52:65-73.

30. An Y, Furber KL, Ji S: Pseudogenes regulate parental gene expression via ceRNA network. J Cell Mol Med 2017, 21(1):185-192.

31. Zhang J, Liu L, Li J, Le TD: LncmiRSRN: identification and analysis of long non-coding RNA related miRNA sponge regulatory network in human cancer. Bioinformatics 2018, 34(24):4232-4240.

32. Wang Y, Zeng X, Wang N, Zhao W, Zhang X, Teng S, Zhang Y, Lu Z: Long noncoding RNA DANCR, working as a competitive endogenous RNA, promotes ROCK1-mediated proliferation and metastasis via decoying of miR-335-5p and miR-1972 in osteosarcoma. Mol Cancer 2018, 17(1):89.

33. Li Z, Jin C, Chen S, Zheng Y, Huang Y, Jia L, Ge W, Zhou Y: Long non-coding RNA MEG3 inhibits adipogenesis and promotes osteogenesis of human adipose-derived mesenchymal stem cells via miR-140-5p. Mol Cell Biochem 2017, 433(1-2):51-60.

34. Lu X, Chen X, Xing J, Lian M, Huang D, Lu Y, Feng G, Feng X: miR-140-5p regulates the odontoblastic differentiation of dental pulp stem cells via the Wnt1/beta-catenin signaling pathway. Stem Cell Res Ther 2019, 10(1):226.

35. Sun DG, Xin BC, Wu D, Zhou L, Wu HB, Gong W, Lv J: miR-140-5p-mediated regulation of the proliferation and differentiation of human dental pulp stem cells occurs through the lipopolysaccharide/toll-like receptor 4 signaling pathway. Eur J Oral Sci 2017, 125(6):419-425.

36. Urbano N, Scimeca M, Crocco A, Mauriello A, Bonanno E, Schillaci O: (18)F-Choline PET/CT Identifies High-Grade Prostate Cancer Lesions Expressing Bone Biomarkers. J Clin Med 2019, 8(10).

37. Manikandan M, Abuelreich S, Elsafadi M, Alsalman H, Almaak H, Siyal A, Hashmi JA, Aldahmash A, Kassem M, Alfayez $M$ et al: NR2F1 mediated down-regulation of osteoblast differentiation was rescued by bone morphogenetic protein-2 (BMP-2) in human MSC. Differentiation 2018, 104:36-41.

38. Cho YD, Yoon WJ, Woo KM, Baek JH, Park JC, Ryoo HM: The Canonical BMP Signaling Pathway Plays a Crucial Part in Stimulation of Dentin Sialophosphoprotein Expression by BMP-2. Journal of Biological Chemistry 2010, 285(47):36369-36376.

39. Hwang S, Park SK, Lee HY, Kim SW, Lee JS, Choi EK, You D, Kim CS, Suh N: miR-140-5p suppresses BMP2-mediated osteogenesis in undifferentiated human mesenchymal stem cells. FEBS Lett 2014, 588(17):2957-2963.

40. Rothman AM, Arnold ND, Pickworth JA, Iremonger J, Ciuclan L, Allen RM, Guth-Gundel S, Southwood M, Morrell NW, Thomas $M$ et al: MicroRNA-140-5p and SMURF1 regulate pulmonary arterial hypertension. J Clin Invest 2016, 126(7):2495-2508.

41. Behr $B$, Leucht $P$, Longaker MT, Quarto N: Fgf-9 is required for angiogenesis and osteogenesis in long bone repair. P Natl Acad Sci USA 2010, 107(26):11853-11858. 
42. Wallner C, Schira J, Wagner JM, Schulte M, Fischer S, Hirsch T, Richter W, Abraham S, Kneser U, Lehnhardt $\mathrm{M}$ et al: Application of VEGFA and FGF-9 enhances angiogenesis, osteogenesis and bone remodeling in type 2 diabetic long bone regeneration. PLOS One 2015, 10(3):e0118823.

43. Wang LP, Roth T, Abbott M, Ho L, Wattanachanya L, Nissenson RA: Osteoblast-derived FGF9 regulates skeletal homeostasis. Bone 2017, 98:18-25.

44. Lu J, Dai J, Wang X, Zhang M, Zhang P, Sun H, Zhang X, Yu H, Zhang W, Zhang L et al: Effect of fibroblast growth factor 9 on the osteogenic differentiation of bone marrow stromal stem cells and dental pulp stem cells. Mol Med Rep 2015, 11(3):1661-1668.

\section{Figures}
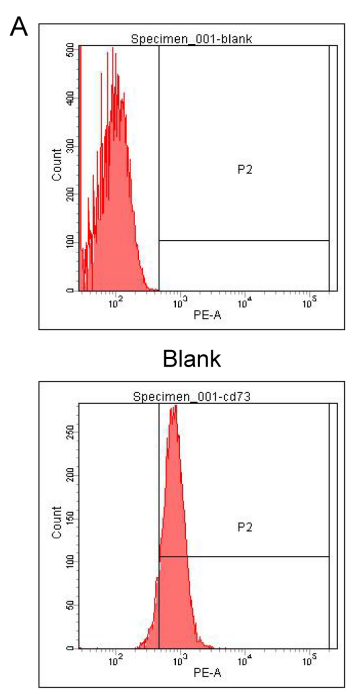

$\operatorname{CD73}(90.7 \%)$

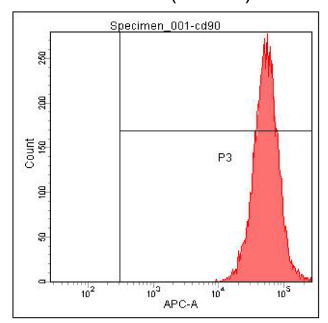

$\operatorname{CD90}(100 \%)$

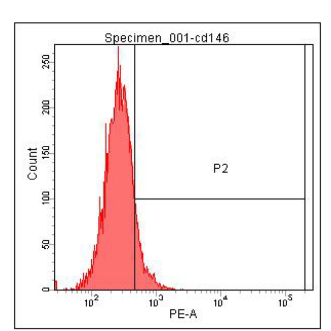

CD146 (10.6\%)

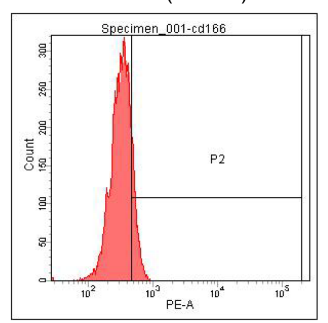

CD166 (12.9\%)

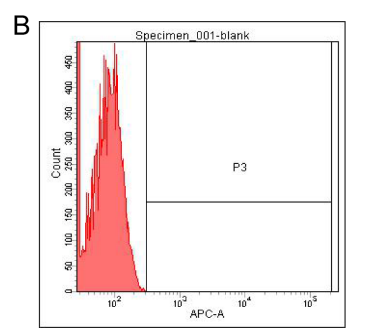

Blank

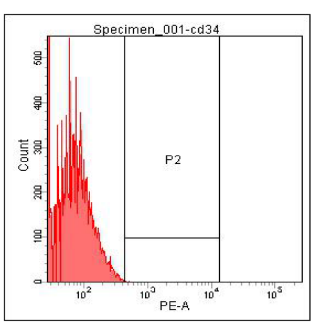

CD34 $(0.1 \%)$

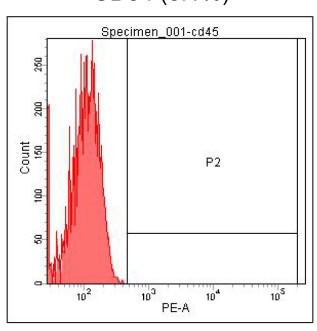

CD45 (0.1\%)
C

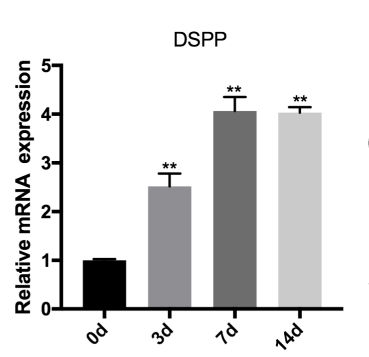

E

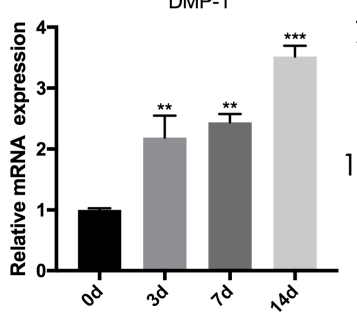

$\mathrm{D}$
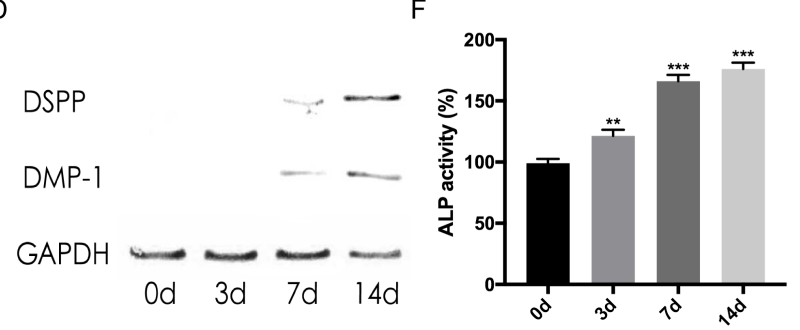

Figure 1

Flow cytometry of hDPSCs and odontoblastic differentiation of hDPSCs after induction for 14 days. A. Representative diagrams are shown for the PE negative control, CD34, CD45, CD73, CD146 and CD166. B. Representative diagrams are shown for the APC negative control and CD90. C-D. The mRNA and protein expression levels of DMP-1 and DSPP increased during odontoblastic differentiation. E. The number of mineralized nodules increased with the process of odontoblastic differentiation. F. The ALP activity of hDPSCs was enhanced after differentiation induction. The data are presented as the mean \pm SD in three independent experiments. ${ }^{*} P<0.05,{ }^{*} \mathrm{P}<0.01,{ }^{\star *} \mathrm{P}<0.001$. 
A

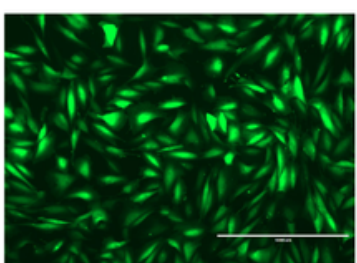

shNC

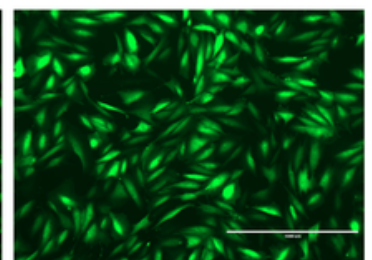

shH19-1

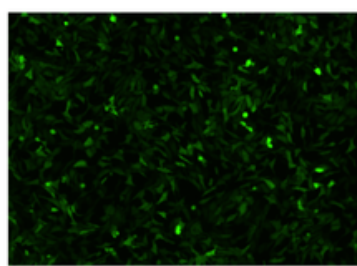

shH19-2
B

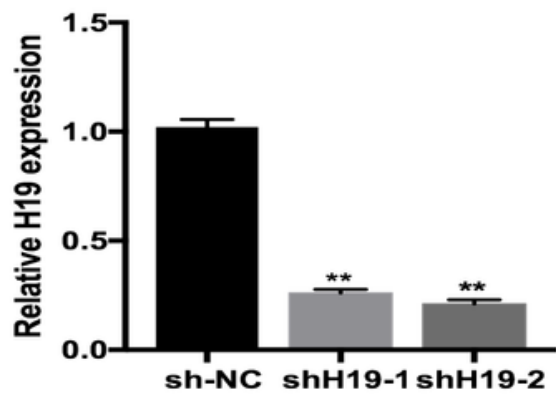

E

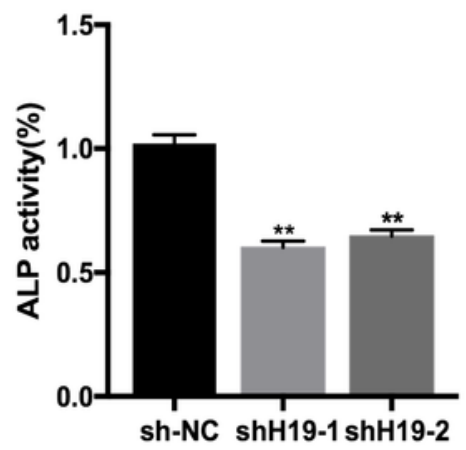

C

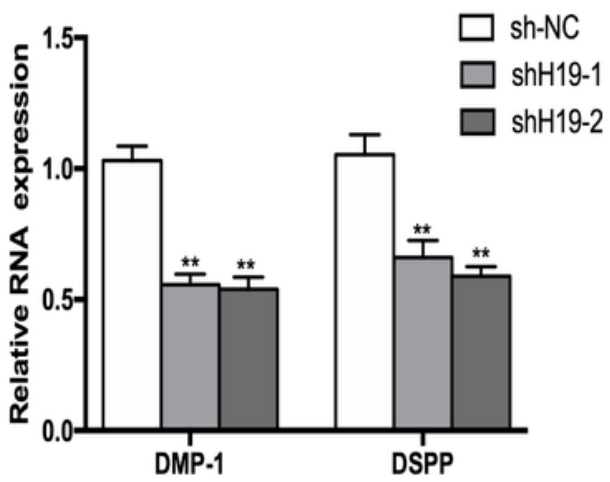

F sh-NC sh-H19-1 sh-H19-2

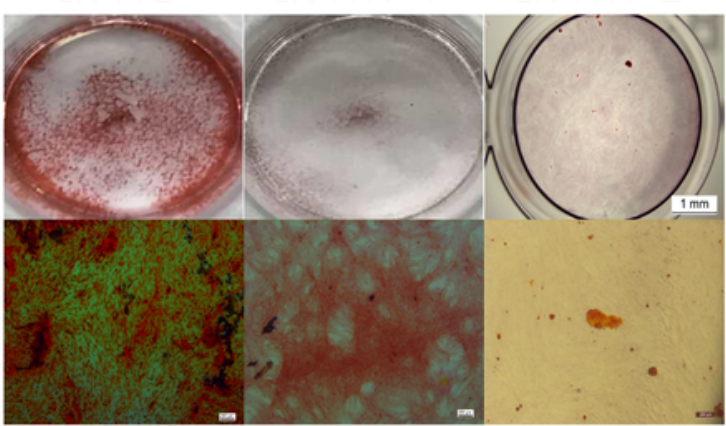

D
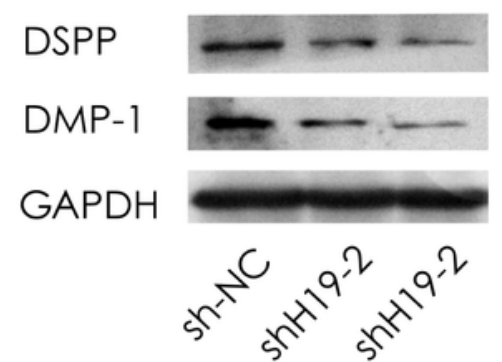

G

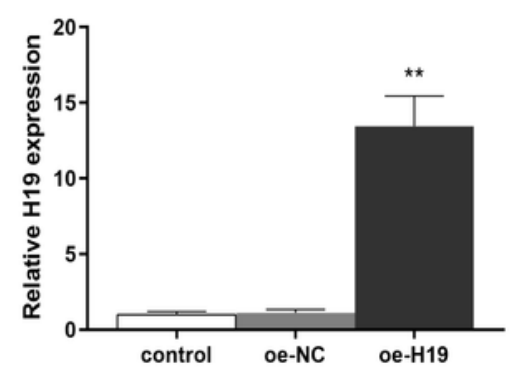

$J$

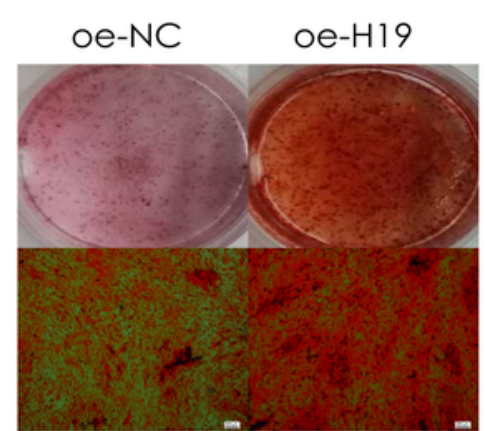

$\mathrm{H}$

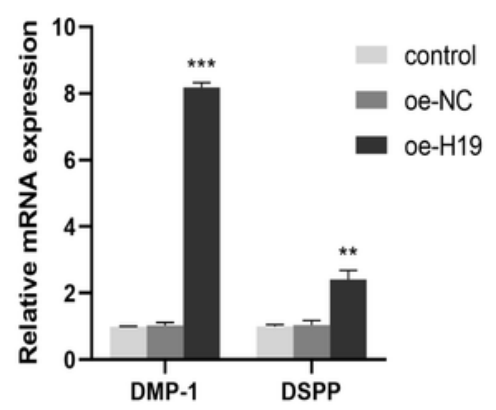

K

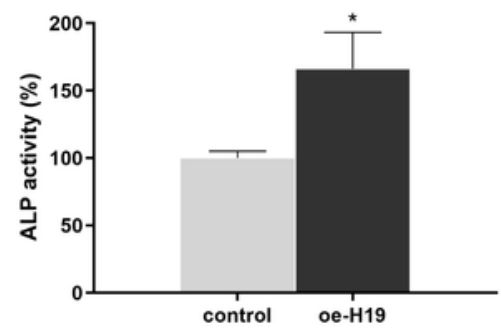

Figure 2

$\mathrm{H} 19$ promotes odontogenesis of hDPSCs. A. Fluorescence was observed under an inverted fluorescence microscope after transfection for 48 hours. B. The expression levels of H19 were determined by qRT-PCR. C. Relative mRNA expressions of DMP-1 and DSPP measured by qRT-PCR after odontoblast induction for 7 days. D. Western blot results showed the protein levels of DSPP and DMP-1 decreased in shH19-1 and shH19-2 groups after odontoblast induction for 7 days. E. The ALP activity was decreased by H19 
knockdown after odontoblast induction for 7 days. F. Images of Alizarin Red S staining in sh-NC, shH19-1, and shH19-2 groups. hDPSCs were cultured in odontoblastic medium for 7 days. G. H19 was upregulated after hDPSCs were transfected with pcDNA 3.1-H19. H. qRT-PCR assay revealed that the mRNA expressions of DMP-1 and DSPP were increased in H19 overexpression group after odontoblast induction for 7 days. I. Western blot analysis of protein expression of DMP-1 and DSPP in hDPSCs after odontoblast induction for 7 days. J. After odontoblast induction for 7 days, Alizarin Red S staining showed that $\mathrm{H} 19$ overexpression group generated more calcified nodules than control group. $\mathrm{K}$. The ALP activity was increased by $\mathrm{H} 19$ overexpression after odontoblast induction for 7 days. The data are presented as the mean $\pm S D$ in three independent experiments. ${ }^{\star} P<0.05,{ }^{\star *} P<0.01,{ }^{\star \star *} \mathrm{P}<0.001$. 
A

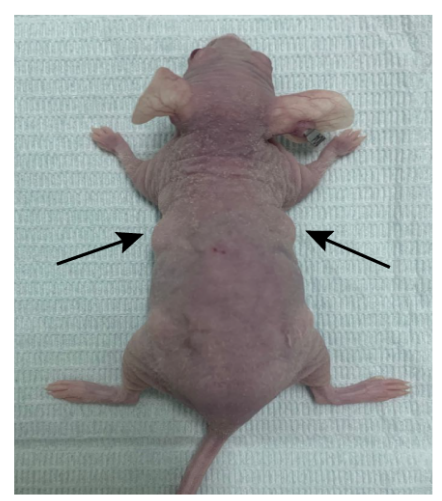

C

H\&E staining

Masson staining

IHC of DMP-1

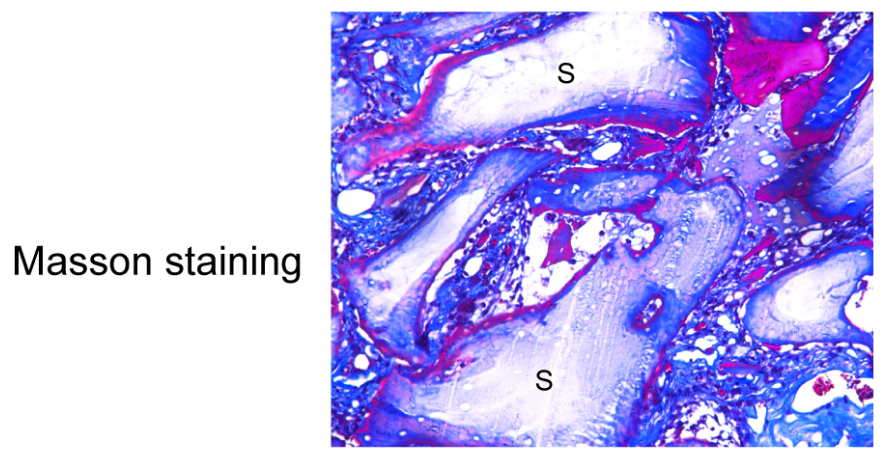

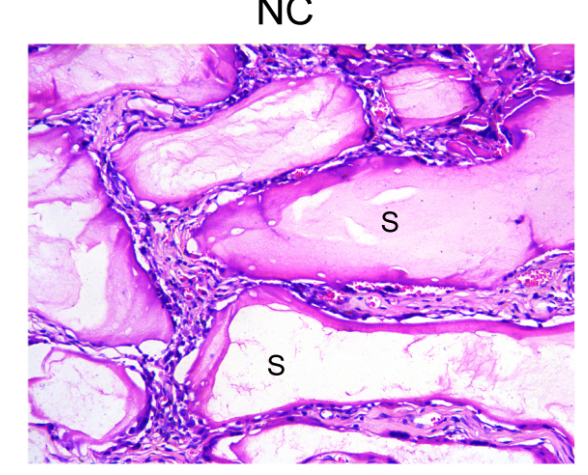

B
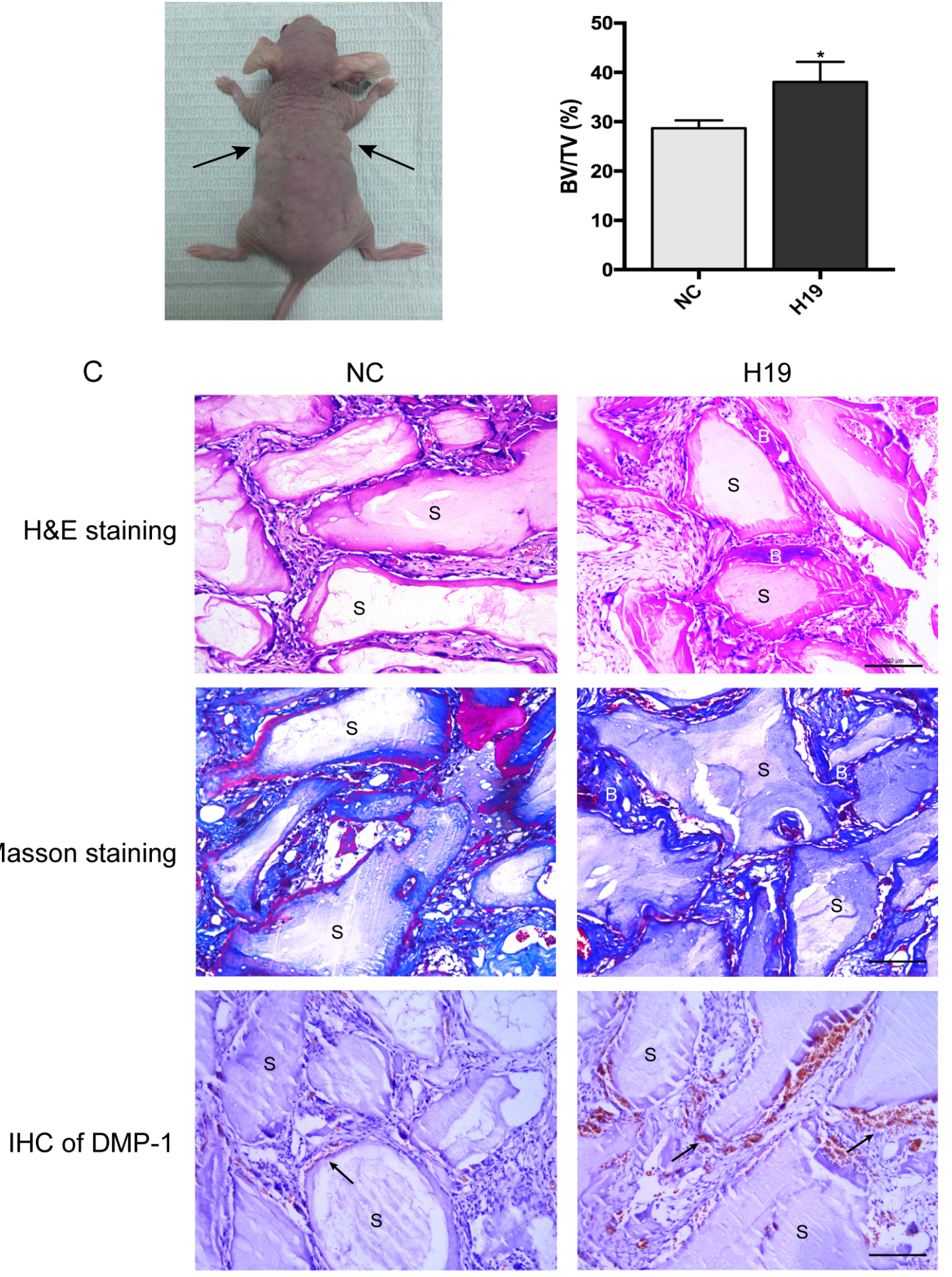

\section{Figure 3}

$\mathrm{H} 19$ enhances the dentinogenesis of hDPSCs in vivo. A. hDPSCs in NC and H19 group were transplanted subcutaneously into 5-week-old BALB/c homozygous nude mice for 6 weeks. B. percentages of new bone volume to tissue volume (BV/TV) of hDPSCs-loaded scaffolds. The data are presented as the mean \pm SD of three experiments. ${ }^{*}<0.05$. C. H\&E staining, Masson staining and immunohistochemical staining of DMP-1 in NC and H19 groups. B: dentin-like tissues, S: scaffold, Scale Bar $=100 \mu \mathrm{m}$. 

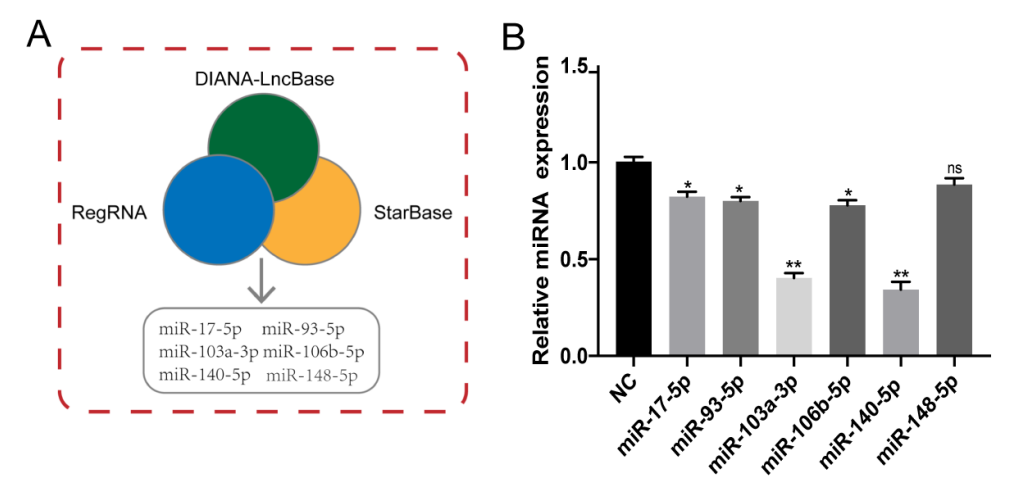

C

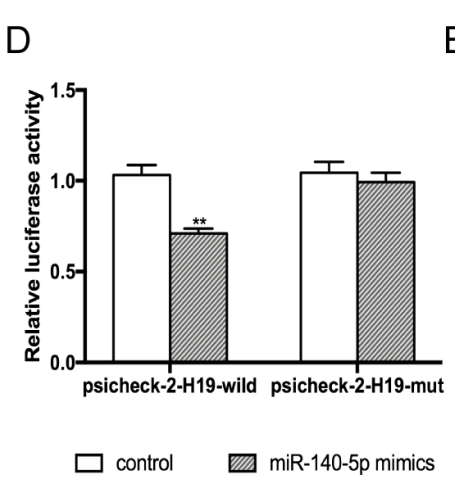

E

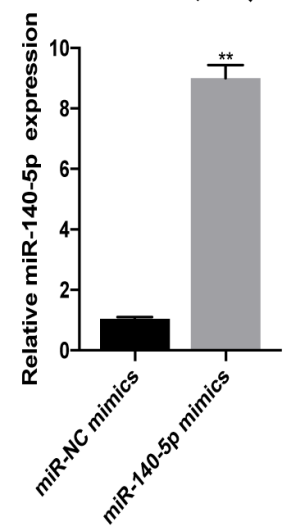

Inc-H19 5'AUGCUGCACUUUACAACCACUG 3' miR-140-5p 3' GAUGGUAUCCCAUUUUGGUGAC 5' Inc-H19 Mut1 5'AUGCUGCACUUCGGCGUACAGA 3'

$F$

OM 7 days

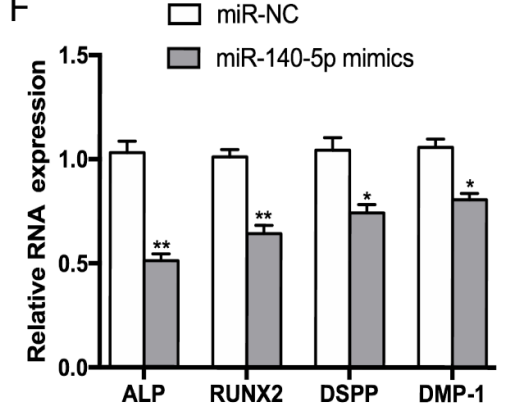

OM 7 days

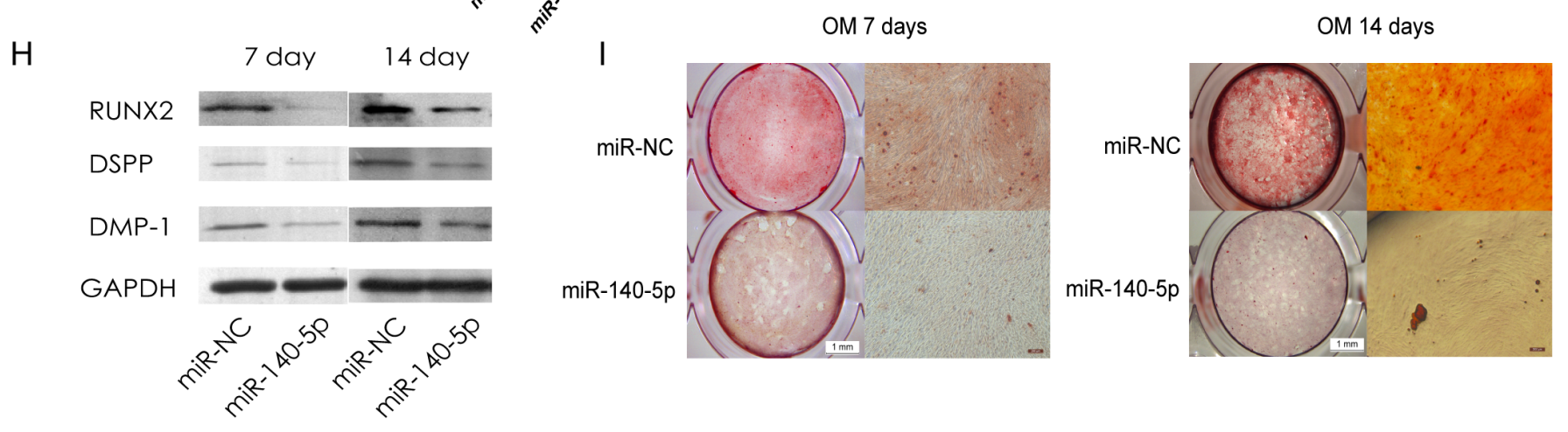

\section{Figure 4}

H19 binds directly to miR-140-5p and overexpression of miR-140-5p inhibits odontoblastic differentiation of hDPSCs. A. Databases consistently predicted six miRNAs interacting with IncRNA H19. B. Relative candidate miRNA expression after odontoblast induction for 14 days compared with that in NC groups. C. Sequence alignment of $\mathrm{H} 19$ with wild type and mutant type miR-140-5p. The potential binding sites were predicted by Starbase. D. Relative luciferase activity of HEK293T cells co-transfected with H19 reporter plasmid and miR-140-5p mimics. E. qRT-PCR analysis was performed to measure the levels of miR-1405p. F. After odontoblast induction for 7 days, relative mRNA expressions of ALP, RUNX2, DSPP and DMP-1 in miR-140-5p mimic group were measured by qRT-PCR. G. Relative mRNA expressions of ALP, RUNX2, DSPP and DMP-1 in miR-140-5p mimic groups and miR-140-5p mimic groups after odontoblast induction for 14 days. H. Relative protein expression of RUNX2, DSPP, and DMP-1 in miR-140-5p mimic transfection groups after odontoblast induction for 7 days and 14 days. I. Representative micrographs of Alizarin Red $S$ staining in miR-140-5p mimic transfection groups after odontoblast induction for 7 days and 14 days. The data are presented as the mean $\pm S D$ in three independent experiments. ${ }^{*} P<0.05,{ }^{*} P<0.01$. 
BMP-2_3'UTR WT 5' UUUAUUUACUAUUAUAACCACUU 3' $\operatorname{miR}-140-5 p$ III IIII

BMP-2_3'UTR Mut 5' UUUAUUUACUAUUAUAACAAACU 3' FGF9_3'UTR WT 5' GCCCUUAAAAAAGUAACCACUA 3' miR-140-5p 3' AUGGUAUCCCAUU- UUGGUGAC 5'
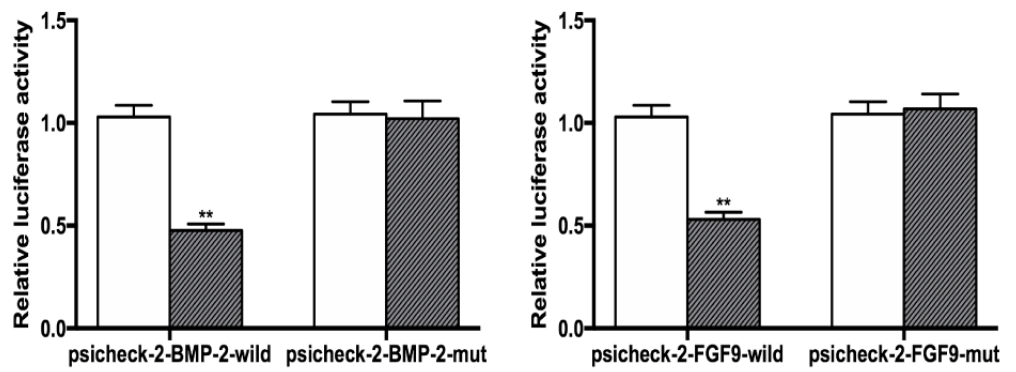
FGF9_3'UTR Mut 5' GCCCUUAAAAAAGUUUGGUGAA 3'

C

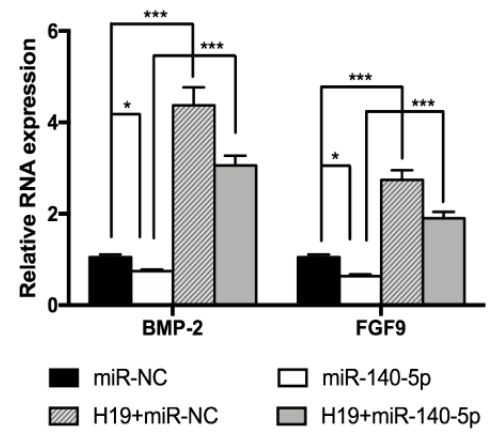

$\mathrm{F}$

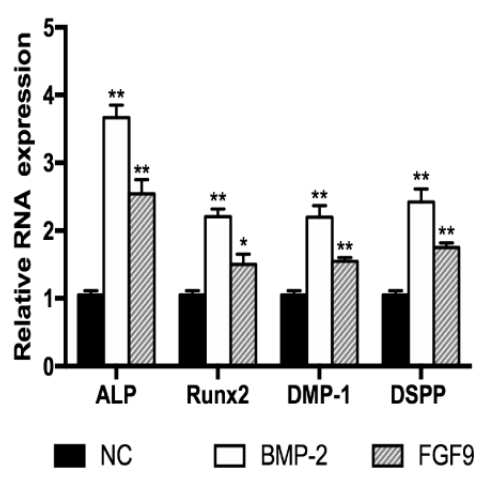

D
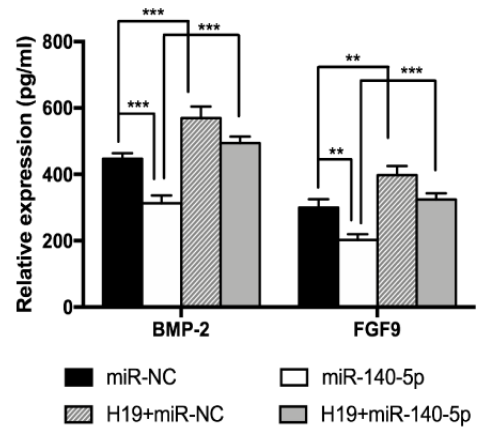

G

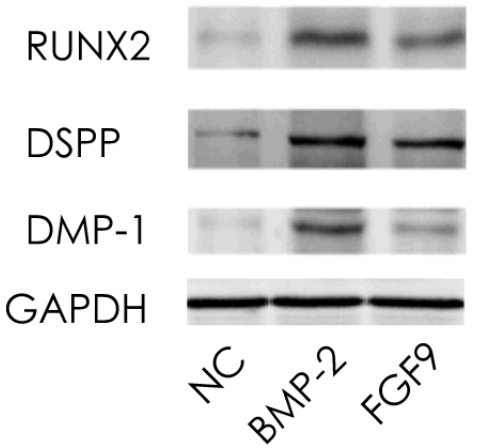

E

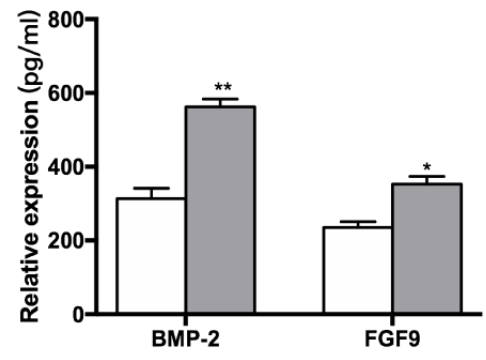

$\square \mathrm{PM} \quad \square \mathrm{OM}$

$\mathrm{H}$

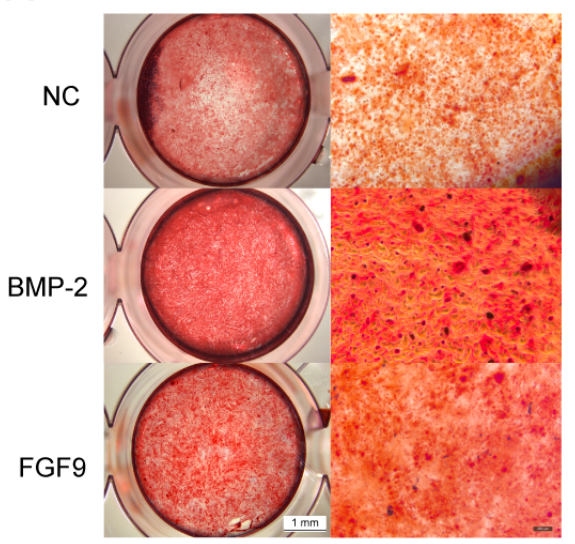

Figure 5

$\mathrm{H} 19$ regulates BMP-2/FGF9 expression by binding to miR-140-5p A. Schematic of BMP-2/FGF9 wild-type (WT) and mutant (Mut) luciferase reporter vectors. B. miR-140-5p significantly reduced luciferase activity in BMP-2/FGF9-wild not in BMP-2/ FGF9-mut. C. Quantification of mRNA expression of BMP-2 and FGF9 measured by qRT-PCR in miR-140-5p mimics, pcDNA3.1-H19, miR-140-5p mimics/pcDNA3.1-H19 cotransfected hDPSCs relative to the control groups. D. Relative expression of BMP-2 and FGF9 in hDPSCs measured by ELISA in miR-140-5p mimics, pcDNA3.1-H19, miR-140-5p mimics/pcDNA3.1-H19 cotransfected hDPSCs. E. Relative expression of BMP-2 and FGF9 in hDPSCs measured by ELISA after odontoblast induction for 14 days. F. Relative mRNA expressions of ALP, RUNX2, DSPP and DMP-1 in exogenous BMP-2 treated groups and exogenous FGF9 treated groups after odontoblast induction for 14 days. G. Relative protein expressions of RUNX2, DSPP and DMP-1 in exogenous BMP-2 treated groups and exogenous FGF9 treated groups after odontoblast induction for 14 days. H. Representative 
micrographs of Alizarin Red S staining in exogenous BMP-2 treated groups and exogenous FGF9 treated groups after odontoblast induction for 14 days. The data are presented as the mean \pm SD in three independent experiments. ${ }^{*} \mathrm{P}<0.05,{ }^{*} \mathrm{P}<0.01,{ }^{\star *} \mathrm{P}<0.001$.
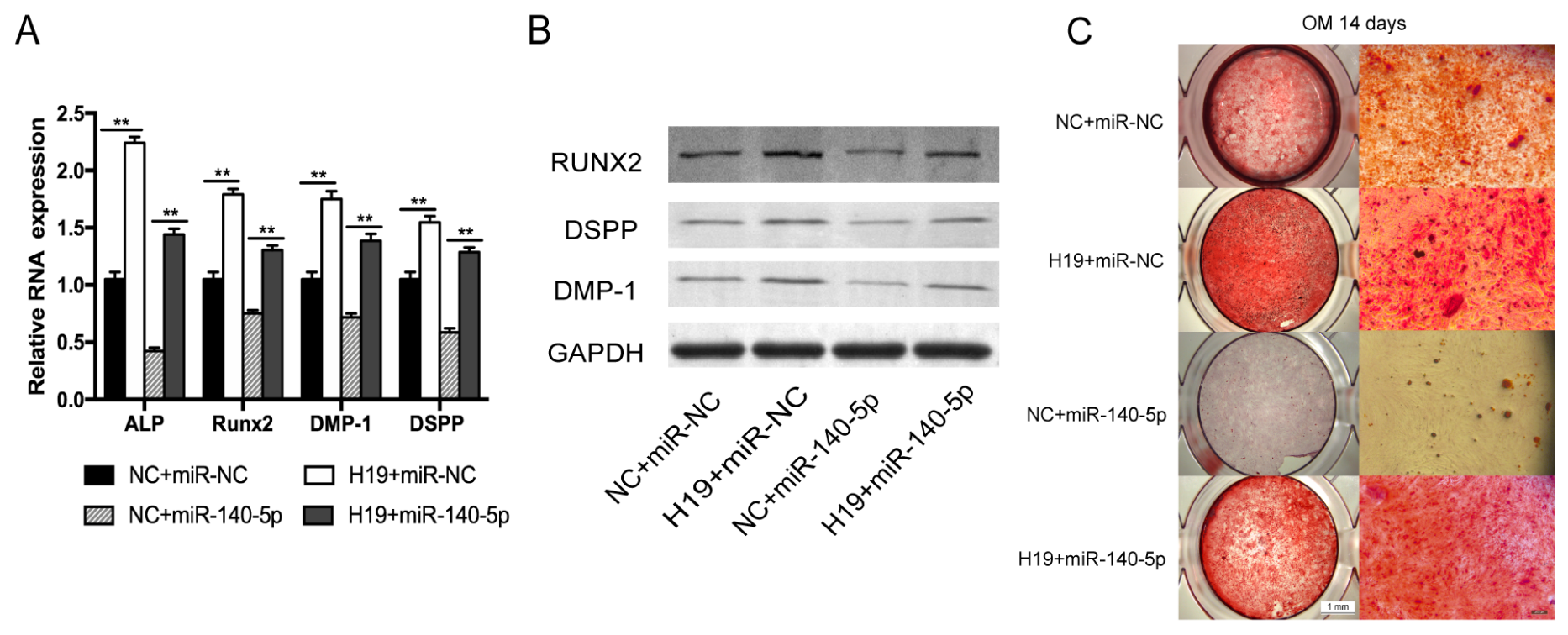

D

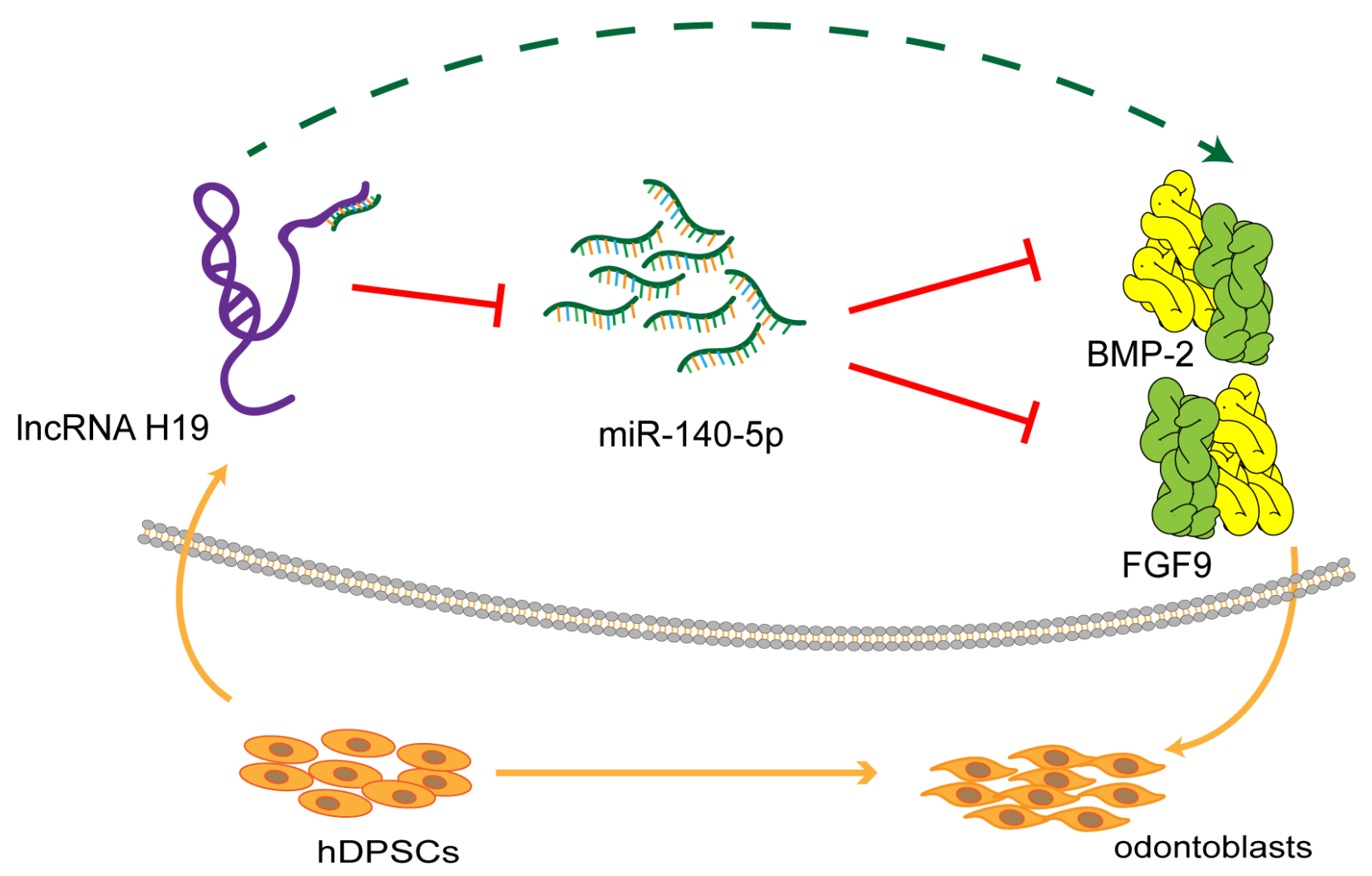

\section{Figure 6}

Overexpression of miR-140-5p reversed the stimulation effect of $\mathrm{H} 19$ in vitro. A. Relative RNA expression of mineralization-related genes in the miR-140-5p co-transfection experiment after odontoblast induction 
for 14 days. B. Relative protein expression of RUNX2, DSPP and DMP-1 in the miR-140-5p co-transfection experiment after odontoblast induction for 14 days. C. Images of Alizarin Red S staining in the miR-140$5 p$ co-transfection experiment. hDPSCs were cultured in odontoblastic medium for 14 days. Histograms revealed quantification of Alizarin Red S staining by spectrophotometry. D. The schematic diagram for IncRNA-H19/miR-140-5p/BMP-2/FGF9 axis. The data are presented as the mean \pm SD in three independent experiments. ${ }^{*} P<0.05,{ }^{\star} * P<0.01$.

\section{Supplementary Files}

This is a list of supplementary files associated with this preprint. Click to download.

- Additionalfile1.doc 